\title{
Möglichkeiten der Digitalisierung zur Beratung von hochkonfigurierbaren und hochindividualisierbaren Gütern
}

\author{
Oliver Blunk • Gordon Brown • Michael Prilla
}

Eingegangen: 21. April 2020 / Angenommen: 9. Juni 2020 / Online publiziert: 19. Juni 2020

(C) Der/die Autor(en) 2020

Zusammenfassung Der Verkauf von hochkonfigurierbaren bzw. hoch-individualisierbaren Gütern ist online aktuell nicht verfügbar, da für den Kunden eine intensive Beratung notwendig ist, um passende Produkte bzw. Konfigurationen zu finden. Zusätzlich stehen Online-Beratungen vor der Herausforderung, dass Kunden unterschiedliche Konfigurationen nicht betrachten oder anfassen können, und der Berater ebenfalls nicht visualisiert wird. Weiterhin verfügen Geschäfte häufig nicht über Ausstellungsstücke, die alle unterschiedlichen Konfigurationsmöglichkeiten abdecken. Basierend auf Literatur und einer eigens in einem Möbelhaus durchgeführten ethnografischen Studie werden Anforderungen an ein System zur Online-Beratung im Kontext der Möbelbranche abgeleitet. Beispielsweise wird untersucht, inwieweit Augmented Reality als Ansatz dabei helfen kann, dass Kunden mit virtuell dargestellten Produkten interagieren können und so unterschiedliche Konfigurationen vergleichen können. Weiterhin werden unterschiedliche Möglichkeiten diskutiert, um den Kunden in diesem Umfeld zu beraten. Basierend auf den Anforderungen und unterschiedlichen Technologien wird ein Szenario erstellt, welches beschreibt, wie eine Online-Beratung durchgeführt werden könnte. Es wird der Einkaufsprozess eines fiktiven Kunden betrachtet. Zusätzlich werden auch Einschränkungen einer online-basierten Beratung diskutiert.

O. Blunk $(\bowtie) \cdot$ G. Brown $\cdot$ M. Prilla

Human-Centered Information Systems, Institut für Informatik, Technische Universität Clausthal, Clausthal-Zellerfeld, Deutschland

E-Mail: oliver.blunk@tu-clausthal.de 
Schlüsselwörter Digitalisierung · Dienstleistung · Personennahe Dienstleistung · Augmented Reality · Beratung

\title{
Potentials offered by digitalizing consultations for highly customizable and configurable products
}

\begin{abstract}
Selling highly customizable goods online is difficult as this often requires intensive consultation in order for customers to find a suitable product and configuration. Furthermore, online consultations entail several difficulties that restrict the customer's experience, such as the absence of visualizations of the desired products and their interaction partner or restricted haptic interactivity with the product. Based on literature and an ethnographic study which we conducted, we derive requirements that would need to be met to solve these problems. Following up, we discuss several options that could serve as means to fulfill these requirements. As an example, we explore using an augmented reality approach to allow customers to interact with virtual products and to inspect different features. Based on the options discussed, we formulate a scenario which details how an online consultation session for furniture sales could be conducted. It follows a fictional customer through their consultation journey from intending to buy online up to the final purchase. Finally, we discuss limitations for conducting consultations online.
\end{abstract}

Keywords Digitalization · Consultation · Service · Augmented Reality

\section{Einleitung}

Obwohl ein Großteil aller Käufe in vielen Produktgruppen und Branchen mittlerweile online durchgeführt wird, existieren immer noch Ausnahmen, wie zum Beispiel hoch-konfigurierbare und -individualisierbare Güter. Bei diesen Produkten ist oft eine intensive Beratung notwendig, da viele Konfigurationsmöglichkeiten existieren und umfangreiches Hintergrundwissen notwendig ist, um eine für Käufer passende Konfiguration zu wählen. Oftmals gibt es vor einem Kauf online die Möglichkeit, sich Bilder oder virtuelle Repräsentationen des Produktes aus verschiedenen Perspektiven anzusehen. Neben diesen existieren auch unterschiedliche Möglichkeiten, sich über Augmented Reality virtuelle Produkte in der eigenen Wohnung anzeigen zu lassen (bspw. App von Ikea). Allerdings ist es in solchen Anwendungen nicht möglich, zeitgleich eine Beratung von einer Fachkraft zu erhalten bzw. aufwändige Konfigurationen von Produkten vorzunehmen. Dies kann von Nachteil sein, da manche Produkte, wie bspw. Möbel oder Wintergärten, erst für den Kunden hergestellt werden müssen und somit über lange Lieferfristen verfügen. Dies stellt für Kunden ein Risiko dar, da ihnen das bestellte Produkt ggf. nach der Lieferung nicht gefallen könnte, weil es bspw. in dem Licht des finalen Stellplatzes anders aussieht, als es auf der Verkaufsplattform angezeigt wurde. Dementsprechend lassen sich solche Güter aktuell schwierig online verkaufen.

Dieser Beitrag beleuchtet die oben beschriebene Problematik am Beispiel der Digitalisierung in der Möbelbranche. Hier ist es dem Kunden bereits im mittleren und 
auch im höheren Preissegment möglich, aus unzähligen Konfigurationsmöglichkeiten zu wählen, was oft eine Beratung notwendig macht. Beispielsweise lassen sich bei aktuellen Couch-Modellen nicht nur Farben und Stoffe konfigurieren, sondern auch unterschiedliche Varianten von (verstellbaren) Arm- und Rücklehnen, Fußund Kopfstützen, Polsterungen, Füßen, Beleuchtungen, etc. Häufig lassen sich auch nicht alle Merkmale frei miteinander kombinieren. Pro Modell entsteht dadurch eine für Endkunden kaum zu überblickende Vielfalt von Konfigurationsmöglichkeiten. Kernkompetenz von Möbelhäusern ist es, Kunden bei der Auswahl aus diesen Möglichkeiten zu beraten. Daher eignet sich die Beratung für Couches in der Möbelbranche als Beispiel für die auftretenden Herausforderungen beim Online-Verkauf hochindividualisierbarer Güter.

In diesem Beitrag werden die folgenden Fragestellungen diskutiert, welche im Zuge der Digitalisierung gelöst werden müssen:

- Wie lassen sich Möbel in einer digitalisierten Beratungslösung zum Kunden bringen, so dass Kunden an der Auswahl aktiv beteiligt werden können?

- Wie lassen sich Beratungsleistungen in einer digitalisierten Beratungslösung zum Kunden bringen?

- Wie lässt sich das digitale Angebot auf viele Kunden skalieren?

Basierend auf Literatur und einer eigenen ethnografischen Studie (Blunk et al. 2020) wird zur Beantwortung der Fragestellungen ein Szenario entworfen. Dieses soll beschreiben, wie der Beratungsprozess digitalisiert und eine digitale Dienstleistung geschaffen werden kann. Zusätzlich wird das Szenario durch Ergebnisse eigener Studien gestützt. Das entwickelte Beratungsszenario gehört zu den personennahen Dienstleistungen (Guerrero et al. 2020), da eine hohe Interaktion (Kellogg and Chase 1995) und auch ein hoher Informationsaustausch bzw. aufgrund mehrerer Beratungstermine (Feldmer et al. 2019) oft längere Interaktionszeiträume vorhanden sind (Parasuraman et al. 1985). Der Hauptbeitrag ist das entwickelte Szenario, was als Grundlage für die Implementierung von Beratungssystemen genutzt werden kann, um die zuvor beschriebenen Probleme bei der Online-Beratung von hochkonfigurierbaren bzw. -individualisierbaren Gütern zu ermöglichen.

\section{Herausforderungen der Digitalisierung}

Zunächst stellt sich die Frage, welche Aspekte einer klassischen Beratung eine wichtige Rolle spielen und deswegen in einer digitalisierten Lösung ebenfalls beachtet werden sollten.

\subsection{Hintergrund}

Eine der größten Herausforderungen der Onlineberatung besteht darin, das Erlebnis für den Kunden, welches dieser im Möbelhaus bei einem normalen Beratungsgespräch haben würde, zufriedenstellend online anzubieten bzw. gegenüber dieser Herangehensweise sogar einen Mehrwert zu bieten. Im Vordergrund steht hierbei der fehlende Verkaufsraum. Dem Kunden wäre es bei einem Beratungsgespräch, welches 
z.B. nur via Text- oder Audiochat durchgeführt wird, nicht möglich, Möbel, welche ihn interessieren könnten, in Lebensgröße und von allen Seiten zu besichtigen. Auch der direkte Vergleich zwischen verschiedenen Varianten eines Möbelstücks fällt an dieser Stelle schwer. Zudem besteht für den Kunden keine Möglichkeit, mit dem Möbelstück zu interagieren: Verfügt eine Couch z. B. über eine ausklappbare Schlaffunktion, kann der Kunde nicht ausprobieren bzw. ansehen, wie die Dimensionen der Couch in der ausgeklappten Form auf einen Raum wirken. Generell wirkt das Fehlen einer haptischen Interaktionsmöglichkeit mit dem Möbelstück negativ auf das Vertrauen in die eigene Fähigkeit, das Produkt einzuschätzen aus und sollte, falls haptische Interaktion nicht möglich ist, durch Alternativen ausgeglichen werden (Peck and Childers 2003). Es muss also eine Visualisierungsmöglichkeit geschaffen werden, welche diesem Umstand entgegenwirkt. Auch das Beratungsgespräch selbst muss in der digitalisierten Lösung durchgeführt und, falls möglich, ergänzt werden. Die Beratung eines Kunden, der zuhause ist, verhindert den gemeinsamen Rundgang durch das Möbelhaus mit dem Berater. Es ist keine Bezugsperson anwesend, Augenkontakt ist nicht möglich und simple Gesten wie das Zeigen auf ein Produktmerkmal ist nicht möglich.

Zudem hat die Nutzung digitaler Hilfsmittel oftmals einen Einfluss auf die Reziprozität, die Beratungsgesprächen entscheidend ist (Dolata and Schwabe 2016): Oft findet in frühen Phasen eines Gesprächs eine Gewöhnungsphase statt, in welcher der Kunde weniger zum Gespräch beiträgt bzw. der Berater das Gespräch leitet, was abschreckend wirken könnte. Dies wirkt sich negativ auf das Beratungsergebnis aus. Eine erhöhte Reziprozität hingegen kann auch das Beratungsergebnis verbessern (Dolata and Schwabe 2016).

Schlussendlich muss die Soziale Präsenz und das Vertrauen des Kunden in den Berater und den Dienstleister gefördert werden, was eine der größten Hürden digitaler Beratungsplattformen darstellt ( $\mathrm{Lu}$ and Fan 2014). Soziale Präsenz kann den Vertrauensbildungsprozesses positiv beeinflussen, während das Vertrauen selbst positive Einflüsse auf das Kaufverhalten des Kunden hat, was auch für digitale Beratungsplattformen gilt (Johnson and Grayson 2005).

Zurzeit existieren unterschiedliche Anwendungen (bspw. von Möbelherstellern wie Ikea), welche die Platzierung von Produkten in Form von 3D-Objekten erlauben. Bisherige Anwendungen erlauben zwar die Platzierung der Produkte, erlauben aber keine aufwändige Konfiguration dieser bzw. ermöglichen keine Beratung durch Fachkräfte. Diese beiden Aspekte sind jedoch notwendig, um individualisierbare bzw. hochkonfigurierbare Güter online zu vertreiben. Dennoch sind existierende Ansätze von Ikea vielversprechend (Alves and Luís Reis 2020). Manche Vorarbeiten haben ein Projektionssystem genutzt, um virtuelle Möbel darzustellen (Khan et al. 2017). Dieser Ansatz ist unter Laborbedingungen gut nutzbar, aber in der Realität schwierig umsetzbar, da Endkunden lernen müssten das Projektionssystems korrekt zu platzieren. Augmented Reality über Head-Mounted-Displays nimmt Endkunden diesen Schritt ab, da die Projektion direkt über die Brille realisiert wird.

Bisherige Literatur konzentriert sich vornehmlich auf die Umsetzung von Konfigurationsmöglichkeiten hinsichtlich der Datenstruktur (Xiong and Wu 2011), was aber den Aspekt der Beratung vernachlässigt. Studien, die sich auf digitalisierte Be- 
ratungsvorgänge in der Möbelbranche konzentrieren, sind aktuell in der Literatur nicht vorhanden.

\subsection{Ergebnisse eigener ethnografischer Arbeiten}

Als Vorarbeit für diesen Beitrag wurde eine ethnografische Studie in einem Möbelhaus durchgeführt (Blunk et al. 2020). Es wurden drei Möbelberater für je einen Tag bei der Arbeit begleitet und beobachtet. Alle drei Teilnehmer hatten bereits mehrjährige Erfahrung in der Möbelberatung. Abschließend wurden semi-strukturierte Interviews geführt. Ziel der Studie war das Erfassen der Domäne des Möbelverkaufs und den dazugehörigen Beratungen, um eine Digitalisierung zu ermöglichen. Im Folgenden werden die Ergebnisse in Form von unterschiedlichen Herausforderungen für Digitalisierungsbestrebungen zusammengefasst.

Die abschließenden Interviews dienen zur Klärung von Rückfragen und Qualifizierung der Beobachtungen. Dies der besseren Einschätzung, ob die beobachteten Abläufe eher eine Ausnahme oder die Norm darstellen. Jeder der drei Berater stand für das abschließende Interview zur Verfügung. Die Interviews dauerten ca. $30 \mathrm{~min}$ und wurden teils durch Kundengespräche unterbrochen. Die Fragen zielten insbesondere auf den Ablauf des Beratungsgesprächs, Kommunikationsabläufe, verwendete Hilfsmittel und die Beschreibung von Anforderungen durch Kunden.

Einerseits gibt es eine Wissensasymmetrie zwischen Beratern (Experten für Möbel) und Kunden (Experten für die eigenen vier Wände) (Jungermann and Fischer 2005). Vor dem Gespräch verfügen beide jeweils nur über allgemeinere Informationen über die Domäne des anderen. Beide versuchen diese Asymmetrie im Rahmen des Gesprächs aufzulösen (Jungermann and Fischer 2005).

Von den Beratern wurde häufig geäußert, dass Kunden nicht ausreichend vorbereitet sind, wobei sich laut einer Marktforschungsstudie ca. $40 \%$ der Kunden vorbereitet fühlen (Feldmer et al. 2019). Kunden fällt es laut den Beratern oft schwer, eigene Wünsche oder Anforderungen zu artikulieren. Ein Grund dafür kann fehlende Übung in der Verbalisierung der Anforderungen an neue Möbel sein, da die meisten Kunden in ihrer Lebenszeit nur wenige Male bspw. eine neue Couch kaufen. Beratern fällt diese Kommunikation andererseits deutlich leichter. Hier ist sicherlich die Erfahrung das eigene Wissen explizit zu machen ein wichtiger Faktor (Smith 2001).

Eine weitere Beobachtung ist die fehlende Vorbereitung von Beratern. Selbst als bekannt war, dass manche Kunden an dem Tag der Beobachtung zwecks Beratung in das Möbelhaus kommen wollten, haben die Berater die Beratung spontan durchgeführt und keine Notizen zur Vorbereitung genutzt. Gemäß der Marktforschungsstudie und laut Aussagen der Berater vergleichen Kunden in mehreren Möbelhäusern Angebote und Preise (Feldmer et al. 2019), weswegen eine intensive Vorbereitung sich nicht in allen Fällen lohnt. Termine zwecks Beratung wurden nicht beobachtet.

In unserer Beobachtung wurden wiederkehrende Kunden konsequent zu den Beratern geschickt, mit denen sie bereits vorher Kontakt hatten. Als Gründe hierfür wurden einerseits die leichtere Verteilung der Provision als auch Vorteile für den Aufbau einer Beziehung zwischen Kunde und Berater genannt, welche für die Beratung förderlich ist. Dies wird auch durch andere Autoren bestätigt (Ingram et al. 2007; Schein 2009). 
Es muss hervorgehoben werden, dass die beobachteten Gespräche sehr durch den Berater dominiert wurden, der als Experte das Gespräch moderiert und maßgeblich vorangebracht hat. Aktive Einbindung des Kunden bzw. die oben beschriebene Reziprozität, die bei Beratungsgesprächen wichtig ist (Dolata and Schwabe 2016), war kaum vorhanden.

Die hier genannten Funde hinsichtlich Wissensasymmetrie, Vorbereitung von Kunden und Beratern und die Kunde-Berater-Beziehung dienen im Folgenden als Grundlage für die Erstellung des Szenarios.

\subsection{Anforderungen}

Zusammenfassend lassen sich aus der Literatur die folgenden Anforderungen ableiten: Interaktion, Reziprozität, Vertrauensbildung und soziale Präsenz. Aus der ethnografischen Studie kommen die Anforderungen hinsichtlich Wissensasymmetrie, Vorbereitung von Kunden und Beratern und die Kunde-Berater-Beziehung hinzu. Im Folgenden werden zu den Anforderungen unterschiedliche Lösungsmöglichkeiten vorgestellt und diskutiert.

Die Anforderung der Visualisierung von konfigurierbaren Produkten innerhalb des eigenen lokalen Kontexts kann über Augmented Reality (AR) gelöst werden. Mit Augmented Reality ist die Überlagerung der Realität mit virtuellen Objekten gemeint. AR basiert häufig auf sogenannten Head Mounted Displays (HMDs). HMDs erlauben dem Nutzer zudem, die Umgebung ohne dazwischengeschaltete Kameras und Bildschirme, wie durch eine reguläre Brille zu betrachten. Die virtuellen Inhalte werden über Prismen in das Sichtfeld des Trägers projiziert. So haben Nutzer, im Gegensatz zu Smartphone-basierten Lösungen, die Hände für Tätigkeiten wie das Zeigen auf bestimmte Bereiche in der eigenen Wohnung oder Produktmerkmale frei, können sich frei um die Möbel bewegen und diese problemlos aus allen denkbaren Perspektiven betrachten. Einige HMDs (bspw. Microsoft HoloLens) können die Umgebung des Kunden digital erfassen und intern als 3D-Modell abspeichern. Dieses 3D-Modell wird genutzt, um virtuelle Inhalte im Raum zu verankern. Damit ist es Kunden möglich, sich auch räumlich um die virtuellen Objekte herum zu bewegen und diese zu betrachten. Gleichzeitig kann AR genutzt werden, damit Kunden mit den Produkten interagieren können. Es können Funktionen der Couches anhand der 3D-Modelle demonstriert werden, wie bspw. die Darstellung einer ausklappbaren Schlaffunktion. Gleichzeitig können Konfigurationen erlebt werden, die aktuell physisch nicht im Verkaufsraum des Möbelhauses vorrätig sind. Kunden sehen direkt das Produkt, was sie später kaufen können an genau der Stelle, an welcher es künftig stehen soll. Für Kunden bietet sich der offensichtliche Mehrwert der Vorschau von Möbelstücken in den eignen vier Wänden vor der Lieferung, welche mehrere Wochen dauern kann. Auch die Retouren-Quote könnte sich mit der Hilfe von Augmented Reality verringern lassen.

Die Anforderung der Reziprozität kann darüber adressiert werden, dass sowohl der Kunde als auch der Berater dazu ermächtigt werden können innerhalb der Beratungsumgebung Anpassungen vorzunehmen. Es könnte bspw. explizit ausgeschlossen werden, dass nur der Berater Farben anpassen kann. Darüber soll der Kunde 
ermächtigt werden, aktiv in die Konfiguration einzugreifen und ein aktiver Akteur in der Beratung zu sein.

Zur Darstellung von menschlichen oder künstlichen Beratern können sich virtuelle Avatare eignen, die in Augmented Reality angezeigt werden. Avatare dieser Art haben sich in der Vergangenheit bereits bewährt, um Kunden ein stärker ausgeprägtes Gefühl der Präsenz (Bente et al. 2004) und des Vertrauens (Komiak et al. 2005) hervorzurufen. Für ein hohes Vertrauen des Kunden sollte gesorgt werden, da dies unter Anderem zu einer erhöhten Akzeptanz des angebotenen digitalen Services führt (Mou et al. 2017) und zu einem besseren Kaufverhalten der Kunden führen kann (Lu and Fan 2014). Für ein stärker ausgeprägtes Gefühl der Präsenz zu sorgen birgt ebenfalls Vorteile, da unter Anderem das Vertrauen des Kunden in den frühen Phasen der Vertrauensbildung positiv beeinflusst wird (Ogonowski et al. 2014). Im Direktvergleich zu reinen Text- und Audio-Lösungen sorgten Avatare in einer Studie für signifikant bessere Bewertungen und lagen ungefähr gleichauf mit einer Audio-Video-Lösung bei der das Bild des Gegenübers übertragen wird (Bente et al. 2008). Dieses Ergebnis lässt sich vor allem durch von Menschen als attraktiv eingestufte Berater-Avatare replizieren (Mull et al. 2015). Auch die (wahrgenommene) Menschlichkeit des Berater-Avatars spielt eine Rolle: Je menschlicher der Avatar ist, desto glaubwürdiger und attraktiver erscheint dieser und desto eher interagieren Menschen mit dem Avatar (Mull et al. 2015). Das Aussehen des BeraterAvatars sollte für optimale Ergebnisse realistisch (Jo et al. 2016) und vom Kunden anpassbar (Hanus and Fox 2015) sein.

Eine weitere Herausforderung ist die Wissensasymmetrie zwischen Kunde und Berater, in welcher der Berater mehr Kenntnisse über Möbel hat und der Kunde sich besser in den eigenen vier Wänden auskennt (Jungermann and Fischer 2005). Das Wissen des Beraters über den lokalen Kontext des Kunden (Einrichtungsstil, Platzbedarfe, Licht, etc.) lässt sich adressieren, indem dieser Zugriff auf die Daten der AR-Brille bekommt: Über Zugriff auf die Kamera in der AR-Brille kann der Berater genau sehen, in welche Richtung der Kunde sieht und wie die Wohnung dort eingerichtet ist. Da die AR-Brille permanent die häusliche Umgebung erfasst, kann der Berater Zugriff auf das erstehende 3D-Modell erhalten. Dadurch erhält er einen guten Überblick über die räumlichen Verhältnisse, wie bspw. Platzangebot und Abstände zu übrigen Möbeln. Dies kann dem Kunden längere Erklärungen des eigenen Kontexts ersparen, dessen Beschreibungen für ihn ungewohnt sind. Das Wissen der Berater lässt sich über erweiterte Informationen in Produktkatalogen an Kunden weitergeben. Darüber können beispielsweise Vor- und Nachteile von bestimmten Polstern erläutert werden. In der ethnografischen Studie wurde beobachtet, dass Kunden oft nicht wussten, dass bei Couches unterschiedliche Farben, Stoffe oder Armlehnen als Konfigurationsmöglichkeiten zur Verfügung standen. Hier können gezielte Hinweise und Hervorhebung an den 3D-Modellen in der Augmented Reality Darstellung den Kunden auf Konfigurationsmöglichkeiten hinweisen.

Wenn Beratung aus der Ferne für Kunden angeboten wird, ergibt sich die Frage, inwieweit die Beratungsdienstleistung für viele Kunden skaliert. Um die Herausforderung der Skalierbarkeit des Systems zu adressieren, können Chatbots genutzt werden, die bei Abwesenheit von Beratern oder bei Beantwortung von Fragen assistieren können. Bei Chatbots handelt es sich um autonome Agenten, welche basie- 
rend auf einer Unterhaltung mit dem Kunden lernen, ein Bedarfsprofil erstellen und automatisch dazu passende Produktempfehlungen aussprechen (Miao et al. 2007). Sie haben außerdem die Aufgabe, das Vertrauen des Kunden in sich selbst und den Dienstleister zu fördern, da dies ihre eigene Glaubwürdigkeit erhöht und ebenfalls das Kaufverhalten der Kunden verbessern kann (Papadopoulou et al. 2001). Wenn sie korrekt eingesetzt werden, haben Chatbots einen positiven Einfluss auf das Kaufverhalten von Kunden (Lee 2004; Hildebrand and Bergner 2019). Hierbei muss bedacht werden, dass verschiedene Einflussfaktoren, wie wahrgenommenes Risiko beim Kauf oder die Komplexität des Produkts, die Überzeugungskraft eines Chatbots beeinflussen können (Swaminathan 2003). Weiterhin kann der Chatbot dabei helfen, Fragen zu bestimmten Produktmerkmalen zu beantworten und so helfen, die Wissensasymmetrie anzugleichen (Cui et al. 2017).

Über gezielte geführte Dialoge oder weitergehende Informationen zu bestimmten Produktmerkmalen kann der Kunde mehr über die Auswahl lernen. Auf diese Weise kann der Kunde das System nutzen, um den Produktbestand zu explorieren und sich auf das Gespräch vorzubereiten. Gleichzeitig kann der Chatbot innerhalb der Beratungsumgebung den Nutzer bspw. darauf hinweisen, dass dieser zunächst das verfügbare Platzangebot sichten sollte oder darüber nachdenken sollte, wie viele Personen regelmäßig auf der Couch sitzen werden. Er kann zudem nachfragen, ob die Personen eher sitzend oder liegend die Couch nutzen, um den Kunden anzuregen, über den eigenen Bedürfnisse nachzudenken, bevor dieser in ein Beratungsgespräch geht. Gleichzeitig können die Antworten des Benutzers auf diese Fragen und auf andere Eingaben in das Beratungssystem für den Berater aufbereitet werden, damit sich dieser auf das Gespräch vorbereiten kann.

\section{Szenario zur Digitalisierung zur Beratung von hochkonfigurierbaren Gütern}

Zur Umsetzung einer technischen Lösung zur Unterstützung des Beratungsprozesses wurde ein Szenario entworfen, welches im Folgenden konkreter beschrieben wird.

\subsection{Szenario zur digitalisierten Beratungsleistung}

Der Kunde möchte eine neue Couch kaufen und hat bisher nur eine grobe Vorstellung von dem, was er kaufen möchte. Er besucht für den Kauf online die Webseite des Systems. Der Kunde kann dort ein Kundenkonto anlegen, in dem seine Beratungshistorie gespeichert wird. Dort hat er die Möglichkeit, den existierenden Katalog an Produkten anzuschauen. Nachdem er erste Produkte gesehen hat, speichert er eine erste Auswahl in seinen persönlichen Favoriten. Um sich die Couch vor einem Kauf in den eigenen vier Wänden anzuschauen, bestellt sich der Kunde vom Anbieter eine AR-Brille nach Hause.

Nach Ankunft der AR-Brille setzt der Kunde diese auf. Die AR-Brille erstellt automatisch ein 3D-Raummodell der Räumlichkeiten, um 3D-Modelle akkurat platzieren zu können. Der Kunde meldet sich mit seinem Konto auf der AR-Brille an und kann darüber auf unterschiedliche Funktionen zugreifen. Die Funktionen werden auf der 
AR-Brille ebenfalls durch einen Chatbot unterstützt, sofern zu dem Zeitpunkt kein menschlicher Berater zugeschaltet ist. Das System bittet den Kunden zunächst, den verfügbaren möglichen Stellplatz für die neue Couch auszumessen. Über die ARBrille markiert der Kunde eine Fläche auf dem Boden, was die Vorauswahl von passenden Couches anhand ihrer Maße bereits eingrenzt. Anschließend kann der Kunde bisherige Favoriten platzieren oder den existierenden Katalog durchsuchen.

Der Kunde möchte nach weiteren Couches stöbern und nutzt die Filterfunktionen auf der Brille. Über das System sind bereits die vorherigen Anfragen (helle Couches für mehrere Personen) und die Abmessungen des Stellplatzes bekannt. Zusätzlich definiert der Kunde auf der AR-Brille, dass er eine Eck-Couch kaufen möchte. Er wählt mehrere Couches für einen engeren Vergleich aus. Ab diesem Zeitpunkt möchte der Kunde jedoch von einem menschlichen Berater unterstützt werden.

Der Kunde verbindet sich daraufhin mit einem menschlichen Berater des Möbelhauses, welcher die Konfiguration der Couch unterstützen soll (siehe auch Abb. 1). Es wird eine Audio-/Videoverbindung erzeugt, sodass beide Personen direkt miteinander sprechen können. Der Berater arbeitet von einem typischen Bildschirmarbeitsplatz im Möbelhaus aus. Er wird für den Kunden als menschlicher Avatar dargestellt, den der Berater fernsteuern kann, um sich bspw. im Sichtfeld des Kunden als Ansprechpartner zu positionieren. Der Berater sieht über die in der AR-Brille integrierte Kamera die häusliche Umgebung des Kunden und die aktuelle Einrichtung als Live-Video und virtualisiert als 3D-Modell. Darüber kann der Berater zusätzliche spezifische Vorschläge für mögliche Modelle machen. Der Kunde spricht mit dem Berater kurz über seine Ideen und Vorstellungen. Über ein zusätzliches Panel kann der Kunde die übrigen Couches, die in die Auswahl übernommen wurden, platzieren. Sowohl der Berater als auch der Kunde können eine platzierte Couch konfigurieren (bspw. Stoffe etc.). Die im Wohnzimmer platzierte virtuelle Couch wird dabei kontinuierlich aktualisiert und reflektiert stets den aktuellen Stand der Konfiguration. Der Berater hat die Möglichkeit auf bestimmte Merkmale der Couch oder Positionen im Raum zu zeigen, was der Avatar als Bewegung widerspiegelt.

Der Kunde kann jederzeit die Beratung unterbrechen und später fortsetzen, falls er bspw. er über eine Konfiguration nachdenken möchte. Das System ermöglicht Kunden und Beratern, asynchron die Konfiguration weiterzuentwickeln.
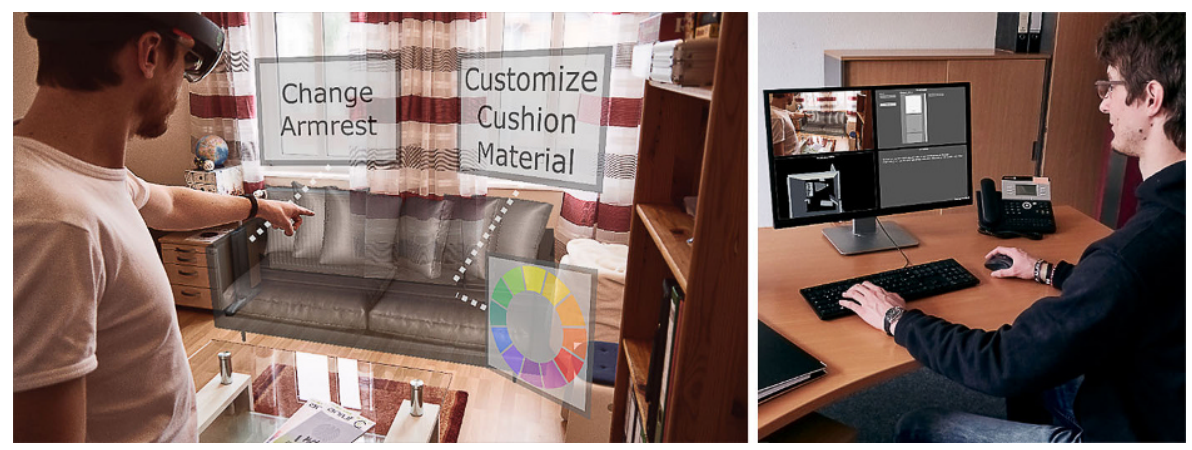

Abb. 1 Die Illustration zeigt, wie ein Kunde (links) eine virtuelle Couch mithilfe von unterschiedlichen Konfigurationsmöglichen anpassen kann. Der Berater (rechts) kann aus der Ferne unterstützen 
Falls der Kunde einfache Fragen außerhalb einer Beratung durch eine Fachkraft stellen möchte, kann er dazu den Chatbot nutzen, der sowohl auf der Webseite des Systems als auch in der AR-Beratungslösung zur Verfügung steht. Bei dem Chatbot kann der Nutzer gezielt persönliche Vorlieben eingeben, um das Angebot an Couches zu filtern oder sich bei bestimmten Vorgängen helfen lassen (bspw. dem Ausmessen des Platzangebots in den eigenen vier Wänden). Der Chatbot soll in der Lage sein, vage Beschreibungen zu verstehen und unterschiedliche Pfade für eine Beratung anzubieten.

Sobald der Kunde mit seiner Auswahl zufrieden ist, kann dieser zusammen mit dem Berater die Beratung beenden und die konfigurierte Couch kaufen.

\subsection{Verknüpfung der Anforderungen mit dem Szenario}

In diesem Abschnitt werden die anfänglich gestellten Forschungsfragen für diesen Beitrag adressiert und es wird beschrieben, wie diese Fragen im Szenario unterstützt werden sollen.

\subsubsection{Wie lassen sich Möbel in einer digitalisierten Beratungslösung zum Kunden bringen, so dass Kunden an der Auswahl aktiv beteiligt werden können?}

In dem beschriebenen Szenario wird Augmented Reality verwendet, um dem Kunden einen virtuellen Katalog von Möbeln anzubieten, aus dem dieser Produkte auswählen kann. Auf diese Weise kann der Nutzer die Couch erleben und diese interaktiv konfigurieren und sich unterschiedliche Merkmale anschauen. Darüber kann der Kunde auch die Wirkung in den eigenen vier Wänden erleben. Über die Darstellung in AR kann der Kunde sogar Konfigurationen der Couch betrachten, die aktuell nicht Verkaufsraum des im Möbelhauses vorhanden sind, wodurch diese Lösung deutliche Mehrwerte gegenüber dem traditionellen Ansatz hat. Damit der Kunde nicht über die Dauer des Beratungsgesprächs ein Tablet oder das Smartphone hochhalten muss, um die virtuelle Couch sehen zu können, wird im Szenario eine AR-Brille eingesetzt.

\subsubsection{Wie lassen sich Beratungsleistungen in einer digitalisierten Beratungslösung zum Kunden bringen?}

Im Szenario wird der Berater auf zwei unterschiedlichen Ebenen adressiert. Zum einen wird ein Avatar genutzt, um einen menschlichen Berater während eines Beratungsgesprächs in $\mathrm{AR}$ zu repräsentieren, welcher dann virtuell im Wohnzimmer des Kunden steht. Der Avatar im Szenario spiegelt ebenfalls die Aktionen, die der Berater tätigt (bspw. das Zeigen auf ein Merkmal der Couch), wodurch eine höhere Präsenz des Beraters erzeugt werden soll (Harms and Biocca 2004; Bente et al. 2008). Zum anderen steht jederzeit ein Chatbot zur Verfügung, der auch Fragen außerhalb der Geschäftszeiten beantworten kann und bei einfachen Konfigurationsaufgaben assistieren kann. 


\subsubsection{Wie lässt sich das digitale Angebot auf viele Kunden skalieren?}

Die Zielsetzung der aktuellen Digitalisierungsbestrebungen ist nicht das Ersetzen menschlicher Berater, sondern eher die Erweiterung der Zugänge zu Beratung, damit auch bei einem Anstieg der Anzahl von Kunden in einem Onlinesystem weiterhin eine qualitative Beratung sichergestellt werden kann. Im Szenario wird dazu ein Chatbot verwendet. Vorteile dieses Ansatzes sind die Skalierbarkeit und der flexible Einsatz in unterschiedlichen Situationen. Der Chatbot soll einerseits bereits auf der Webseite genutzt werden, damit der Kunde erste Informationen zu Couches erhalten kann und andererseits auch in dem Augmented Reality System, damit der Kunde auch ohne menschlichen Berater Hilfestellung bei der Benutzung und geeignete Vorschläge erhalten kann.

\section{Auf dem Weg zu einer digitalisierten Dienstleistung}

Über das skizzierte Szenario lässt sich bereits eine skalierbare Beratungsdienstleistung abbilden, über die Kunden und Berater gemeinsam oder alleine eine Konfiguration eines Möbelstücks vornehmen können. Während die Nutzung von AR bspw. auf Mobiltelefon-Apps zur Anzeige von Möbeln größerer Hersteller und Verkäufer bereits in der Praxis nutzbar ist, stellt es nach Kenntnis der Autoren den ersten Ansatz dar, in dem diese Technologie ein immersives Erlebnis zu Hause mit der Bereitstellung skalierbarer Beratungsleistungen verbindet.

In Abb. 2 wird die aktuelle Implementierung des Szenarios gezeigt. Der Kunde trägt hier eine AR-Brille und kann darüber sowohl virtuelle Möbel (oben links) als auch den virtuellen Berater (oben rechts) sehen. Darüber hinaus kann der Kunde das Aussehen seiner virtuellen Möbelstücke konfigurieren (oben links).

Dennoch gibt es weitere Aspekte, die im Szenario noch nicht ausreichend abgedeckt sind. Eine Herausforderung besteht darin, dass es dem Berater nicht möglich ist, den Kunden im Onlineberatungsgespräch zu beobachten. Somit ist es ihm auch nicht möglich, auf Reaktionen des Kunden zu bestimmten Vorschlägen oder der Beratungsrichtung zu reagieren oder dem Kunden basierend auf Beobachtungen zu deren körperlicher Verfassung Vorschläge zu unterbreiten. Hier werden weitere Studien zeigen müssen, inwieweit die Stimme der Kunden für Beratungsleistungen ausreichend sein kann.

Berater sind gewöhnt eine Person während einer Beratung physisch gegenüber zu stehen und Funktionen von Couches direkt an einem Ausstellungsstück demonstrieren zu können. Dies ist in einer digitalisierten Beratungsdienstleistung nicht mehr möglich. Dementsprechend ändert sich auch die berufliche Identität des Beraters und der Berater muss über die nötige Flexibilität verfügen und in die Lage versetzt werden, um seine persönlichen Beratungskompetenzen auch digital umsetzen zu können (Brown et al. 2012).

Eine weitere Herausforderung ist die für Kunden fehlende Möglichkeit der haptischen Probe, welche einer Umfrage ca. 60\% der Kunden vor einem Kauf wünschen (Feldmer et al. 2019). Aktuelle Online-Anbieter ermöglichen das Versenden von Stoffproben. Eine Nachbildung von den Stoffen (Degraen et al. 2019) oder speziel- 

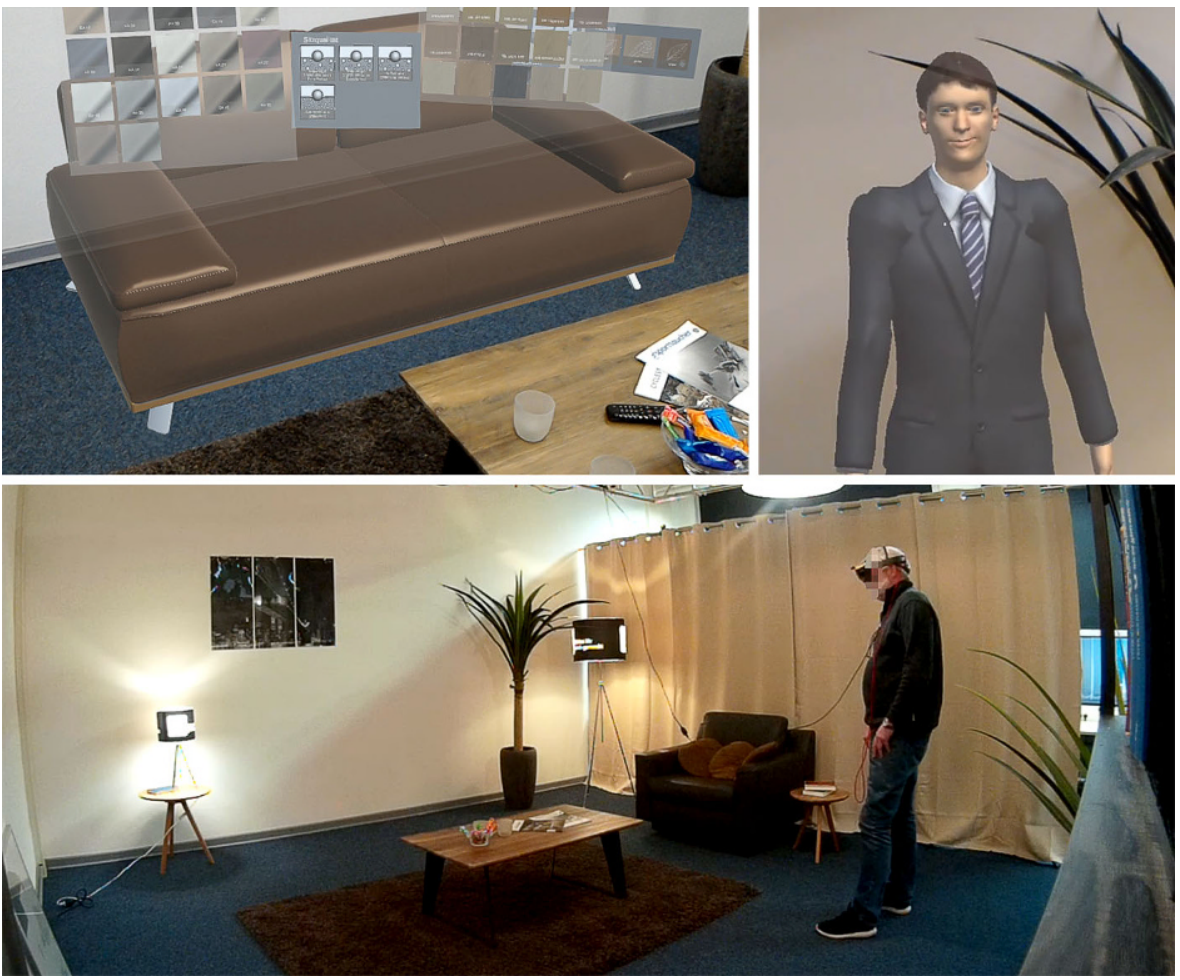

Abb. 2 Unten ist ein Proband gezeigt, der im Möbellabor eine virtuelle Couch platziert hat, welche der Kunde konfigurieren kann (oben links). Der Berater ist als Avatar symbolisiert (oben rechts) und für den Kunden sichtbar

le Handschuhe sind alternativ denkbar (Piekarski and Thomas 2003). Eine weitere Möglichkeit ist der Aufbau einer Datenbank, welche bevorzugte Stoffe o. Ä. des Kunden aus früheren Möbelhaus-Besuchen sammelt und diese mit der aktuellen digitalen Auswahl vergleicht, sodass der Kunde mit einer Referenz arbeiten kann.

\section{Zusammenfassung}

Der vorliegende Beitrag zeigt Herausforderungen bei der Digitalisierung existierender ,konventioneller“ Beratungsdienstleistungen am Beispiel der Möbelbranche. Es werden Anforderungen an Beratungsgespräche aus der Literatur und einer eigenen ethnografischen Studie hergeleitet. Für diese Anforderungen werden unterschiedliche Lösungsmöglichkeiten diskutiert. Basierend auf den Lösungsvorschlägen wird ein Szenario entworfen, welches beschreibt, wie eine digitale Beratungsdienstleistung in der Möbelbranche online durchgeführt werden kann. Das vorgestellte Szenario lässt sich vermutlich auf andere Domänen übertragen, in denen ebenfalls hochkonfigurierbare bzw. -individualisierbare Güter mit Hilfe von Beratungen verkauft werden, wie bspw. Wintergärten, Autos oder Trainings an komplexen Maschinen. 
Wir haben bereits erste Studien durchgeführt, um die Umsetzbarkeit des Szenarios zu evaluieren. Darunter war eine Studie, die analysiert, inwieweit unterschiedliche Avatare für Berater einen Unterschied auf die Beratung machen oder welche Form der Beratungsumgebung für Berater (bspw. Videoübertragung des HMD oder eine VR-Umgebung des 3D-Modells des Wohnzimmers) hilfreich sein können. Die ersten Ergebnisse dieser Studien sind vielversprechend und zeigen, dass das Szenario bisher eine gute Ausgangslage bietet (Brown und Prilla 2020; Kahrl et al. 2020). Daher sind wir optimistisch, dass das Szenario einen guten Ansatz zur Digitalisierung der Beratungsleistung darstellt. Weitere Studien sind notwendig, um unterschiedliche Aspekte des Szenarios zu untersuchen.

Danksagung Dieser Beitrag ist Teil des ARBAY-Projekts (http://arbay-projekt.de), welches durch das BMBF gefördert wird. Wir danken allen Kollegen für die wertvolle Zusammenarbeit.

Funding Open Access funding provided by Projekt DEAL.

Open Access Dieser Artikel wird unter der Creative Commons Namensnennung 4.0 International Lizenz veröffentlicht, welche die Nutzung, Vervielfältigung, Bearbeitung, Verbreitung und Wiedergabe in jeglichem Medium und Format erlaubt, sofern Sie den/die ursprünglichen Autor(en) und die Quelle ordnungsgemäß nennen, einen Link zur Creative Commons Lizenz beifügen und angeben, ob Änderungen vorgenommen wurden.

Die in diesem Artikel enthaltenen Bilder und sonstiges Drittmaterial unterliegen ebenfalls der genannten Creative Commons Lizenz, sofern sich aus der Abbildungslegende nichts anderes ergibt. Sofern das betreffende Material nicht unter der genannten Creative Commons Lizenz steht und die betreffende Handlung nicht nach gesetzlichen Vorschriften erlaubt ist, ist für die oben aufgeführten Weiterverwendungen des Materials die Einwilligung des jeweiligen Rechteinhabers einzuholen.

Weitere Details zur Lizenz entnehmen Sie bitte der Lizenzinformation auf http://creativecommons.org/ licenses/by/4.0/deed.de.

\section{Literatur}

Alves C, Luís Reis J (2020) The intention to use E-commerce using augmented reality-the case of IKEA place. In: Rocha Á, Ferrás C, Montenegro MCE, Medina García VH (Hrsg) Information technology and systems. Springer, Cham, S 114-123

Bente G, Rüggenberg S, Krämer NC, Eschenburg F (2008) Avatar-mediated networking: increasing social presence and interpersonal trust in net-based collaborations. Hum Commun Res 34:287-318. https:// doi.org/10.1111/j.1468-2958.2008.00322.x

Bente G, Rüggenberg S, Krämer NC (2004) Social Presence and Interpersonal Trust in Avatar-Based, Collaborative Net-Communications. In: Alcañiz Raya M (Hrsg) Seventh Annual International Workshop Presence 2004. UPV, Valencia, S 54-61

Blunk O, Brown G, Osmers N, Prilla M (2020) Potentials of AR technology for the digitalization of consultancy intensive sales processes on the example of furniture sales. In: Gronau N, Heine M, Krasnova H, Pousttchi K (Hrsg) Entwicklungen, Chancen und Herausforderungen der Digitalisierung. GITO, Postdam, S 57-72

Brown A, Bimrose J, Barnes S-A, Hughes D (2012) The role of career adaptabilities for mid-career changers. J Vocat Behav 80:754-761. https://doi.org/10.1016/j.jvb.2012.01.003

Brown G, Prilla M (2020). The Effects of Consultant Avatar Size and Dynamics on Customer Trust in Online Consultations. Proc Mensch und Computer 2020 (im Druck)

Cui L, Huang S, Wei F et al (2017) Superagent: a customer service Chatbot for E-commerce websites. In: Proceedings of ACL 2017 System Demonstrations. Association for Computational Linguistics, Vancouver, S 97-102 
Degraen D, Zenner A, Krüger A (2019) Enhancing texture perception in virtual reality using 3D-printed hair structures. In: Proceedings of the 2019 CHI Conference on Human Factors in Computing Systems. ACM, New York, S 249:1-249:12

Dolata M, Schwabe G (2016) More interactivity with IT support in advisory service encounters? In: Gesellschaft für Informatik e.V. (Hrsg) Mensch und Computer 2016-Tagungsband

Feldmer S, Turtur B, Waburek R (2019) Superwohnstudie. http://www.superwohnstudie.de/. Zugegriffen: 12.06.2020

Guerrero R, Lohrenz L, Lattemann C, Robra-Bissantz S (2020) Digitalisierung (und Automatisierung) personennaher Dienstleistungen - eine bibliometrische Analyse. In: Bruhn M, Hadwich K (eds) Automatisierung und Personalisierung von Dienstleistungen. Gabler Verlag (im Druck)

Hanus MD, Fox J (2015) Persuasive avatars: the effects of customizing a virtual salesperson's appearance on brand liking and purchase intentions. Int J Hum Comput Stud 84:33-40. https://doi.org/10.1016/j. ijhcs.2015.07.004

Harms C, Biocca F (2004) Internal consistency and reliability of the networked minds measure of social presence

Hildebrand C, Bergner A (2019) AI-driven sales automation: using chatbots to boost sales. NIM Mark Intell Rev 11:36-41. https://doi.org/10.2478/nimmir-2019-0014

Ingram TN, LaForge RW, Avila RA, et al (2008) Professional selling: a trust-based approach, 4th ed. Thomson/South-Western, Mason, $\mathrm{OH}$

Jo D, Kim K-H, Kim GJ (2016) Effects of avatar and background representation forms to co-presence in mixed reality (MR) tele-conference systems. In: SIGGRAPH ASIA 2016 virtual reality meets physical reality: modelling and simulating virtual humans and environments ACM, S 12

Johnson D, Grayson K (2005) Cognitive and affective trust in service relationships. J Bus Res 58:500-507. https://doi.org/10.1016/S0148-2963(03)00140-1

Jungermann H, Fischer K (2005) Using expertise and experience for giving and taking advice, S 157-173

Kahrl N, Prilla M, Blunk O (2020). Show me your Living Room: Investigating the Role of Representing User Environments in AR Remote Consultations. Proc Mensch und Computer 2020 (im Druck)

Kellogg DL, Chase RB (1995) Constructing an empirically derived measure for customer contact. Manag Sci 41:1734-1749. https://doi.org/10.1287/mnsc.41.11.1734

Khan MF, Ahsan K, Hussain MA (2017) Virtual Reality Projection Based Furniture Showroom - A Hypothetical Study of BPR Implementation in a Small Business of Furniture Design \& Supplier. Bahria Univ J Inf Commun Technol 10:1-13

Komiak S, Wang W, Benbasat I (2005) Comparing customer trust in virtual salespersons with customer trust in human salespersons. In: Proceedings of the 38th annual Hawaii international conference on system sciences. IEEE, Big Island, S 175a

Lee W-P (2004) Towards agent-based decision making in the electronic marketplace: interactive recommendation and automated negotiation. Expert Syst Appl 27:665-679. https://doi.org/10.1016/j.eswa. 2004.07.001

Lu B, Fan W, Zhou M (2016) Social presence, trust, and social commerce purchase intention: An empirical research. Comput Hum Behav 56:225-237. https://doi.org/10.1016/j.chb.2015.11.057

Miao C, Yang Q, Fang H, Goh A (2007) A cognitive approach for agent-based personalized recommendation. Knowl Based Syst 20:397-405. https://doi.org/10.1016/j.knosys.2006.06.006

Mou J, Shin D-H, Cohen JF (2017) Trust and risk in consumer acceptance of e-services. Electron Commer Res 17:255-288. https://doi.org/10.1007/s10660-015-9205-4

Mull I, Wyss J, Moon E, Lee S-E (2015) An exploratory study of using 3D avatars as online salespeople: the effect of avatar type on credibility, homophily, attractiveness and intention to interact. J Fash Mark Manag Int J 19:154-168. https://doi.org/10.1108/JFMM-05-2014-0033

Ogonowski A, Montandon A, Botha E, Reyneke M (2014) Should new online stores invest in social presence elements? The effect of social presence on initial trust formation. J Retail Consum Serv 21:482-491. https://doi.org/10.1016/j.jretconser.2014.03.004

Papadopoulou P, Andreou A, Kanellis P, Martakos D (2001) Trust and relationship building in electronic commerce. Internet Res 11:11

Parasuraman A, Zeithaml VA, Berry LL (1985) A conceptual model of service quality and its implications for future research. J Mark 49:41-50. https://doi.org/10.1177/002224298504900403

Peck J, Childers TL (2003) To have and to hold: the influence of Haptic information on product judgments. J Mark 67:35-48. https://doi.org/10.1509/jmkg.67.2.35.18612

Piekarski W, Thomas BH (2003) Tinmith-mobile outdoor augmented reality modelling demonstration. In: The Second IEEE and ACM International Symposium on Mixed and Augmented Reality, 2003. Proceedings, S 317-318 
Schein EH (2009) Helping: how to offer, give, and receive help. Berrett-Koehler Publishers, San Francisco Smith EA (2001) The role of tacit and explicit knowledge in the workplace. J Knowl Manag 5:311-321. https://doi.org/10.1108/13673270110411733

Swaminathan V (2003) The impact of recommendation agents on consumer evaluation and choice: the moderating role of category risk, product complexity, and consumer knowledge. J Consum Psychol 13:93-101. https://doi.org/10.1207/S15327663JCP13-1\&2_08

Xiong XQ, Wu ZH (2011) The framework of information collection and data management for mass customization furniture. Adv Mater Res 317-319:88-92. https://doi.org/10.4028/www.scientific.net/ AMR.317-319.88 